\title{
General continuum model for twisted bilayer graphene and arbitrary smooth deformations
}

\author{
Leon Balents ${ }^{1,2 \star}$ \\ 1 Kavli Institute for Theoretical Physics, University of California, \\ Santa Barbara, CA 93106-4030 \\ 2 Canadian Institute for Advanced Research, Toronto, Ontario, Canada \\ $\star$ balents@kitp.ucsb.edu
}

\begin{abstract}
We present a simple derivation of a continuum Hamiltonian for bilayer graphene with an arbitrary smooth lattice deformation - technically in a fashion parametrized by displacement fields with small gradients. We show that this subsumes the continuum model of Bistritzer and Macdonald for twisted bilayer graphene [1] as well as many generalizations and extensions of it. The derivation is carried out entirely in real space.
\end{abstract}

(c) (1) Copyright L. Balents.

This work is licensed under the Creative Commons

Attribution 4.0 International License.

Published by the SciPost Foundation.
Received 05-09-2019

Accepted 01-10-2019

Published 10-10-2019

\section{Contents}

1 Introduction 1

2 Effective field theory and a local description $\quad 2$

3 Construction of the continuum model $\quad 3$

3.1 Transformation to global coordinates 3

3.2 Intra-layer energetics $\quad 5$

$\begin{array}{lll}3.3 & \text { Interlayer tunneling } & 6\end{array}$

$\begin{array}{ll}3.4 & \text { Application to a rigid twist } \\ \end{array}$

4 Conclusion $\quad 9$

$\begin{array}{lr}\text { References } & 10\end{array}$

\section{Introduction}

The experimental discovery of correlated insulators and superconductivity in magic angle Twisted Bilayer Graphene (TBG) [2,3] has galvanized experimental research on these structures [4-10]. The bedrock for theory in these systems is the seminal work by Bistritzer and 
Macdonald [1] (BM), who developed a continuum model (CM) for TBG, and by solving it, predicted the presence of magic angles at which the bands closest to the Fermi energy become anomalously flat. The CM achieves several key simplifications over microscopic lattice models. First, it restores periodicity, enabling the application of Bloch's theorem to TBG, which is, strictly speaking, generally only a quasiperiodic system. Second, it reduces the two basic dimensionless parameters of the problem - the ratio of interlayer to intralayer hopping, and the twist angle - to a single one.

The CM of BM has been the basis for most theoretical studies of TBG, and has been elaborated and extended in various ways. Notably, the effect of lattice relaxation is believed to be quantitatively important in isolating the flat bands from those further from the Fermi energy near the first magic angle [11]. More generally, non-ideal strains and inhomogeneity are increasingly studied both theoretically and experimentally [12-14]. Strong coupling of the acoustic phonon modes of graphene to TBG has also been discussed within the CM as a mechanism for superconductivity and linear-in-temperature resistivity in the normal state [15-18].

The derivation by BM is based on an explicit calculation of the overlap matrix elements between quasi-momentum states in the two nearby graphene layers for rigidly rotated sheets, carried out entirely in momentum space. Transformed back into real space, they obtain an intuitive form of spatially dependent inter-layer hopping. Later works either follow the same procedure or simply lift the earlier result. Further effects have been added incrementally, e.g. external strains in Ref. [14].

In the present paper, we present a path to obtain BM's model directly in real space, which we hope has conceptual value in its economy. To do so, we view the twist of the bilayer as a special case of a displacement gradient, following the classical theory of elasticity. We show that any deformation described by an inhomogeneous displacement field (with a small gradient) in each layer can be treated with accuracy equal to that of the case of a uniform small angle twist. In this way, we obtain a model for arbitrary elastic deformations of the sheets, which applies beyond TBG, and indeed subsumes other generalizations of the BM model.

\section{Effective field theory and a local description}

To proceed, we recall the reasoning which justifies the envelope function approximation in semiconductors, or more generally the applicability of continuum effective field theory. The general principles of the latter are that, first: the continuum description of a perturbed system applies when the perturbations are weak and slowly varying on the lattice scale. Second: the continuum Hamiltonian consists of the integral of a local Hamiltonian density which is an analytic function of the perturbations and expressable in an expansion in these perturbations and in small gradients. In our case we view the ideal graphene layer as the unperturbed system and the inter-layer coupling and elastic deformation of the layers as perturbations.

Consider a general elastic deformation of a single graphene layer. The textbook description is through the displacement field $\tilde{\boldsymbol{u}}(\boldsymbol{R})$ defined so that the location of the point $\boldsymbol{R}$ in the undeformed solid becomes $x$ with

$$
x=R+\tilde{u}(R) .
$$

The purpose of the tilde on the displacement field will be revealed shortly. The deformation is locally small if $\partial_{\mu} \tilde{\boldsymbol{u}} \ll 1$, and elasticity theory applies. However, this does not mean that $\tilde{\boldsymbol{u}}(\boldsymbol{R})$ is small, and indeed we do not wish to make this assumption (it is invalid specifically in the most interesting case of a layer rotation).

The case of large displacements makes the use of the $\tilde{\boldsymbol{u}}(\boldsymbol{R})$ field problematic, because the argument $\boldsymbol{R}$ of $\tilde{\boldsymbol{u}}$ no longer represents the location of a point in physical space. When we consider two layers with different large deformations, a single value of $\boldsymbol{R}$ corresponds to two 
very different physical locations, so that locality is not manifest in these variables. The problem is associated with the "Lagrangian" coordinates adopted in Eq. (1), where the deformation is associated with a point in the undistorted lattice. The solution of this problem is to work instead in "Eulerian" coordinates, in which the displacement field $\boldsymbol{u}(\boldsymbol{x})$ is associated with an actual location in the deformed solid:

$$
x=R+u(x) .
$$

This equation implicitly defines $\boldsymbol{x}(\boldsymbol{R})$ from $\boldsymbol{u}(\boldsymbol{x})$, and while it may be less familiar to e.g. readers of Ashcroft+Mermin, it has the distinct advantage that $\boldsymbol{u}(\boldsymbol{x})$ actually describes the deformation of the graphene sheet at position $x$. Hence, the effective Hamiltonian density will be a local functional of $\boldsymbol{u}(\boldsymbol{x})$. It may be helpful to point out that the Eulerian displacement field used here is a two-dimensional analog of the phase coordinate of a charge density wave, which describes a one-dimensional periodic structure in a similar Eulerian fashion $[19,20]$.

\section{Construction of the continuum model}

Now we are in a position to derive the CM. It will be expressed in terms of a continuum description of the electrons, i.e. a Dirac field for each valley and layer, as well as a continuum description of the lattice, i.e. an Eulerian displacement field for each layer. There are three physical effects we need to capture:

1. Pure geometry: A displacement changes the physical positions of atoms, and a nonuniform displacement changes lengths. This is particularly important when the displacements of the two layers are different. We must describe the physics in a global laboratory coordinate frame, which necessarily cannot follow that of both layers in that case.

2. Intra-layer energetics: In addition to the above purely geometrical effect, deforming the atoms in a layer (i.e. strain) changes its energetics, for example through the distance dependence of the hopping matrix elements. The physical effect of strain on graphene has been intensively studied, and is understood to be represented by an "artificial gauge field" [21,22], which is equal and opposite for the two graphene valleys (in order that strain preserves time-reversal symmetry).

3. Inter-layer coupling: It is well-known that a small displacement of one layer relative to one another (a fraction of a lattice constant) completely modifies the inter-layer hopping (e.g. changing from $\mathrm{AA}$ to $\mathrm{AB}$ alignment), drastically changing the energy dispersion. We need to keep track of this same physics locally in the bilayer with slowly-varying displacements.

In the following, we will discuss each of these effects in turn, and how they are incorporated into a continuum description.

\subsection{Transformation to global coordinates}

Let us imagine that the tight binding Hamiltonian in each single layer of graphene is completely unmodified, but we associate each unit cell center, ideally at point $R$, with a deformed point at $\boldsymbol{x}$ described by $\boldsymbol{u}(\boldsymbol{x})$ as in Eq. (2). By assumption, in the original coordinates the physics is unchanged, so we may define continuum Dirac fields $\psi_{ \pm}(R)$ in the standard way, with the subscript denoting the valley, via (see e.g. Ref. [22] for a standard analysis deriving the Dirac Hamiltonian and continuum fields)

$$
c(\boldsymbol{R}) \sim \psi_{+}(\boldsymbol{R}) e^{i K \cdot \boldsymbol{R}}+\psi_{-}(\boldsymbol{R}) e^{-i \boldsymbol{K} \cdot \boldsymbol{R}},
$$



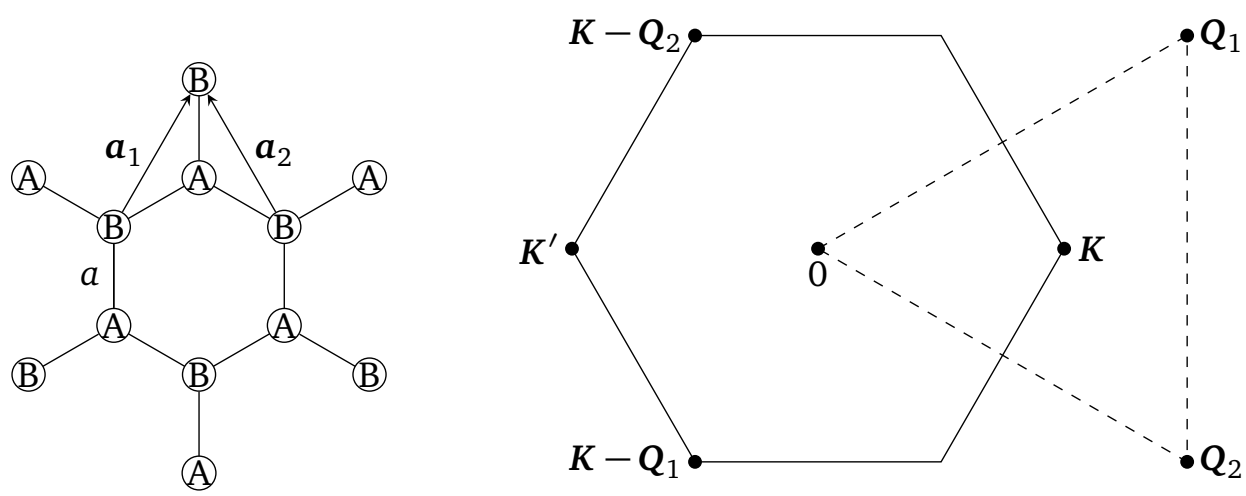

Figure 1: Left panel: conventions for graphene lattice. Two linearly independent Bravais lattice (translation) vectors $\boldsymbol{a}_{1}, \boldsymbol{a}_{2}$ are shown. The distance between neighboring carbon atoms is $a$, as marked. Right panel: Graphene Brillouin zone and some other useful wavevectors. The wavevectors $\boldsymbol{Q}_{1}$ and $\boldsymbol{Q}_{2}$ are basis vectors for the reciprocal lattice. The $K$ point (also denoted $K$ point in the text) is the centroid of the triangle formed by the origin, $\boldsymbol{Q}_{1}$ and $\boldsymbol{Q}_{2}$. The two other Brillouin zone corners $\boldsymbol{K}-\boldsymbol{Q}_{1}$ and $\boldsymbol{K}-\boldsymbol{Q}_{2}$ are equivalent to $\boldsymbol{K}$ as quasimomenta, and are obtained from the latter by $C_{3}$ rotations.

where $K=(4 \pi /(3 \sqrt{3} a), 0)$ ( $a$ is the distance between two carbon atoms) is the zone corner momentum of one valley, see Fig. 1 . We will focus on the $K$ valley, as within the continuum description the valleys do not mix, and henceforth drop the valley index $\psi_{+} \rightarrow \psi$. Results for the other valley are readily derived by $C_{2}$ symmetry. Note that the lack of mixing is an excellent approximation when the displacement gradients are small, because then the slowly-varying nature of the perturbations cannot generate sufficient momentum to mix the two valleys. The Hamiltonian in the original coordinates has the simple Dirac form:

$$
H_{\text {Dirac }}=-i v \sum_{\mu=1,2} \int d^{2} R \psi^{\dagger} \tau^{\mu} \frac{\partial}{\partial R_{\mu}} \psi
$$

where $\tau^{\mu}$ are the Pauli matrices acting in the sublattice space, and $v$ is the Dirac velocity. Now we must transform to the global coordinates $\boldsymbol{x}$. We must determine the transformation property of the Dirac field. To obtain it, we change coordinates in Eq. (3) using Eq. (2) to obtain (again keeping only terms associated with the $K$ valley):

$$
c(\boldsymbol{x})=\left|\operatorname{det}\left(\frac{\partial R_{\mu}}{\partial x_{v}}\right)\right|^{1 / 2} c(\boldsymbol{R}(\boldsymbol{x})) \sim(1-\nabla \cdot \boldsymbol{u})^{1 / 2} \psi(\boldsymbol{R}) e^{i \boldsymbol{K} \cdot(\boldsymbol{x}-\boldsymbol{u}(\boldsymbol{x}))} \equiv \psi(\boldsymbol{x}) e^{i \boldsymbol{K} \cdot \boldsymbol{x}},
$$

where the determinant factor is the usual conformal transformation factor for Dirac fermions, which ensures that the transformed field $\psi(\boldsymbol{x})$ has canonical commutation relations. This implies that

$$
\psi(\boldsymbol{x})=(1-\boldsymbol{\nabla} \cdot \boldsymbol{u})^{1 / 2} \psi(\boldsymbol{R}) e^{-i \boldsymbol{K} \cdot \boldsymbol{u}(\boldsymbol{x})} \leftrightarrow \psi(\boldsymbol{R})=\frac{\psi(\boldsymbol{x})}{(1-\boldsymbol{\nabla} \cdot \boldsymbol{u})^{1 / 2}} e^{i \boldsymbol{K} \cdot \boldsymbol{u}(\boldsymbol{x})} .
$$

Now we can introduce Eq. (6) into the Dirac equation, Eq. (4), and change variables from $\boldsymbol{R}$ to $x$. We require the field transformation and the transformation of the integration measure and of the gradients. The measure is

$$
d^{2} \boldsymbol{R}=d^{2} \boldsymbol{x} \operatorname{det}\left(\frac{\partial R_{\mu}}{\partial x_{v}}\right) \approx d^{2} \boldsymbol{x}(1-\boldsymbol{\nabla} \cdot \boldsymbol{u})
$$


Note that the determinental factor in the field transformation, Eq. (6), cancels against the one from the integration measure, Eq. (7) - this is because the massless Dirac fermion is a conformal field theory. The gradient transforms according to

$$
\frac{\partial}{\partial R_{\mu}}=\frac{\partial x_{v}}{\partial R_{\mu}} \frac{\partial}{\partial x_{v}} \approx \frac{\partial}{\partial x_{\mu}}+\frac{\partial u_{v}}{\partial x_{\mu}} \frac{\partial}{\partial x_{v}} .
$$

Both Eq. (7) and Eq. (8) are given to first order in displacement gradients. Using now Eqs. $(6,7,8)$ in Eq. (4) and keeping terms to linear order in displacement gradients, we obtain finally

$$
H_{\text {Dirac }}=\int d^{2} \boldsymbol{x} \psi_{l}^{\dagger}\left[-i v\left(\tau^{\mu}+\frac{\partial u_{l, \mu}}{\partial x_{v}} \tau^{\nu}\right) \frac{\partial}{\partial x_{\mu}}+v\left(\boldsymbol{K} \cdot \partial_{\mu} \boldsymbol{u}_{l}\right) \tau^{\mu}\right] \psi_{l} .
$$

Here we have introduced a layer index $l$, though the individual layers are decoupled at this point. Note that all the changes in Eq. (9) from Eq. (4) are entirely due to the change of coordinates. Absolutely nothing of the electron dynamics has changed (yet). Successive terms containing $\boldsymbol{u}_{l}$ in Eq. (9) represent: (1) a linear transformation of spin matrices coming from the gradient transformation; and (2), a shift of the Dirac point, coming from the field redefinition. Specifically, demanding that the energy vanish at the location of the shifted Dirac point, we obtain the Dirac point shift

$$
\delta K_{l}=-K_{\mu} \nabla u_{l, \mu}=-K \nabla u_{l, x},
$$

to linear order in displacement gradients (here $K=|K|$ ). Owing to the lack of a derivative action on the Dirac fields in the shift term, this is the most important effect of lattice deformations accounted for in Eq. (9).

\subsection{Intra-layer energetics}

Now we consider the physical effects of deforming the graphene layers on the energetics within each layer. This is the problem of single layer graphene with strain, which has been extensively studied since the early work of Ando in the context of carbon nanotubes in 2002 [21]. The effect of the strain is to induce an "artificial gauge field" $\mathcal{A}$ which enters the Dirac Hamiltonian

$$
H_{\mathrm{agf}}=\int d^{2} \boldsymbol{x} \psi_{l}^{\dagger} v \mathcal{A}_{l} \cdot \tau \psi_{l} .
$$

Here we can express the gauge field in terms of strain as

$$
\mathcal{A}_{l}=\frac{\gamma}{a}\left(\begin{array}{c}
\epsilon_{x x}^{l}-\epsilon_{y y}^{l} \\
2 \epsilon_{x y}^{l}
\end{array}\right),
$$

where $\gamma$ is an order one constant proportional to $\partial \ln t / \partial \ln a$ - the logarithmic derivative of the hopping strength with respect to bond length - and $\epsilon^{l}$ is the strain tensor in layer $l$,

$$
\epsilon_{\mu \nu}^{l}=\frac{1}{2}\left(\partial_{\mu} u_{l, \nu}+\partial_{\nu} u_{l, \mu}\right) .
$$

The artificial gauge field here is that of the $K$ valley. Of of opposite sign appears for the $K^{\prime}$ valley, thereby maintaining time-reversal symmetry.

Comparing to Eq. (9), we see that the artificial gauge field is of the same magnitude as the $K$-point shift, because both contributions are proportional to a single gradient of displacement, with a coefficient of order the Dirac velocity divided by the microscopic lattice spacing (note $K \propto 1 / a$ ). So, for a generic strained layer, this is an important effect. However, for a rigid rotation, the strain tensor vanishes and so does the artificial gauge field. Hence, if the rotation is not too inhomogeneous, we can neglect the strain effect in this section completely. 


\subsection{Interlayer tunneling}

What remains is to consider the coupling between layers. We will assume that the main coupling mechanism, at the single particle level, is tunneling between layers, a standard assumption, made e.g. by BM [1]. We proceed to determine the form of the tunneling contribution using a symmetry analysis. In general, the tunneling contribution to the Hamiltonian can be written as

$$
H_{\text {tun }}=\int d^{2} \boldsymbol{x} \psi_{2}^{\dagger} \mathrm{T}\left(\boldsymbol{u}_{1}-\boldsymbol{u}_{2}\right) \psi_{1}+\text { h.c., }
$$

where $\boldsymbol{T}(\boldsymbol{u})$ is a $2 \times 2$ matrix in the sublattice space, which depends upon the difference $\boldsymbol{u} \equiv \boldsymbol{u}_{1}-\boldsymbol{u}_{2}$ of the displacement fields. In writing Eq. (14) we have used several principles. First, we have assumed spin-independent tunneling, which is justified by the negligible spin-orbit coupling in graphene. Next, we have assumed that the tunneling term is local in the continuum model, as is the standard assumption in effective field theory. Furthermore, we neglected any dependence upon the gradients of the displacements. The latter is certainly present, but sub-dominant. By the standard analyticity assumption of effective field theory, $H_{\text {tun }}$ is an analytic function of the gradients, and because they are assumed small, $H_{\text {tun }}$ is dominated by its zeroth order term. To be a bit more precise, we are assuming there are two small parameters: the tunneling strength and the size of the displacement gradients. We are keeping terms in the Hamiltonian linear in these parameters, but not higher order ones.

Notably, unlike the effects in the previous two subsections, the tunneling energy in Eq. (14) depends explicitly on the displacements themselves (and not only on their gradients). The single layer terms could not be functions of the displacements directly, because the energy of a single layer must be independent of a uniform displacement of that layer. The interlayer energy can and does depend upon their relative displacement. However, it must be independent of a uniform and simultaneous translation of both layers, which is why it is a function only of the difference $\boldsymbol{u}_{1}-\boldsymbol{u}_{2}$.

To further constrain the tunneling matrix, we take into account remaining symmetries. First, we have a remaining discrete translational symmetry: the energy must be independent of a constant displacement of one layer (relative to the other) by a Bravais lattice vector. This implies the condition

$$
\mathrm{T}(\boldsymbol{u}+\boldsymbol{a})=\mathrm{T}(\boldsymbol{u}), \quad \boldsymbol{a} \in \text { B.L. }
$$

Consequently, we can represent the tunneling matrix in a Fourier series

$$
\mathrm{T}(\boldsymbol{u})=\sum_{\mathbf{Q} \in R . L .} \mathrm{T}_{\mathbf{Q}} e^{i \mathbf{Q} \cdot u}
$$

where the sum is over reciprocal lattice vectors, and $T_{Q}$ are Fourier coefficients.

There are a few remaining symmetries to use. We consider here only those symmetries which preserve the valley - others serve to determine the Hamiltonian for the other valley from this one. In applying these symmetries, we take the origin of the un-displaced lattice to lie at the center of a hexagon, and both layers to be aligned directly atop one another. The symmetry operations are:

1. $C_{2}$ T: The combination of time-reversal symmetry with a two-fold rotation through the hexagon center (of the un-displaced lattice).

2. $R_{y}: y \rightarrow-y$ reflection through a plane cutting mid-way through a carbon-carbon bond,

3. $C_{3}$ rotation about the center of a hexagon (of the un-displaced lattice). 
Note that the operations are symmetries only when both the electrons and the displacements are transformed, because any given configuration of displacements typically is not invariant under the operations.

To use these symmetries, we need to understand how they act on the fields $\psi_{l}(\boldsymbol{x})$ and $\boldsymbol{u}_{l}(\boldsymbol{x})$. The transformations of $\boldsymbol{u}_{l}$ are same as those of the coordinates, as it is a vector, and those of $\psi$ can be inferred by demanding the invariance of Eq. (9). Putting this together, one finds that under $C_{2} \mathrm{~T}$,

$$
C_{2} T: \quad \psi_{l}(\boldsymbol{x}) \rightarrow \mathcal{C} \tau^{x} \psi_{l}(-\boldsymbol{x}), \quad \boldsymbol{u}_{l}(\boldsymbol{x}) \rightarrow-\boldsymbol{u}_{l}(-\boldsymbol{x}) .
$$

Here $\mathcal{C}$ is the complex conjugation operator. Under the $y$ reflection,

$$
R_{y}: \quad \psi_{l}(x, y) \rightarrow \tau^{x} \psi_{l}(x,-y), \quad \boldsymbol{u}_{l}(x, y) \rightarrow \mathrm{O}_{2} \boldsymbol{u}_{l}(x,-y)
$$

where $\mathrm{O}_{2}=\left(\begin{array}{cc}1 & 0 \\ 0 & -1\end{array}\right)$. Finally, the $C_{3}$ rotation is the most non-trivial:

$$
\begin{aligned}
C_{3}: & \psi_{l}(\boldsymbol{x}) \rightarrow e^{i \boldsymbol{Q}_{1} \cdot \boldsymbol{u}_{l}\left(\boldsymbol{x}^{\prime}\right)} e^{2 \pi i \tau^{z} / 3} \psi_{l}\left(\boldsymbol{x}^{\prime}\right), \quad \boldsymbol{u}_{l}(\boldsymbol{x}) \rightarrow \mathrm{O}_{3}^{-1} \boldsymbol{u}_{l}\left(\boldsymbol{x}^{\prime}\right), \\
& \boldsymbol{x}^{\prime}=\mathrm{O}_{3} \boldsymbol{x},
\end{aligned}
$$

with

$$
\mathrm{O}_{3}=\left(\begin{array}{cc}
-\frac{1}{2} & \frac{\sqrt{3}}{2} \\
-\frac{\sqrt{3}}{2} & -\frac{1}{2}
\end{array}\right) .
$$

Here $\boldsymbol{Q}_{1}$ is one of the shortest graphene reciprocal lattice vectors (see Fig. 1 and Eq. (27)), and $\mathrm{O}_{3}$ is a $C_{3}$ rotation matrix. The $e^{2 \pi i \tau^{z} / 3}$ factor in front of the transformed $\psi$ in Eq. (19) ensures that as the coordinate $x$ is rotated, the sublattice Dirac matrices follow. The exponential of the displacement field compensates for the rotation of the $K$ point (at the "eastern" corner of the Brillouin zone) to its equivalent point (at the "southwestern" corner), which is separated from its original position by $\boldsymbol{Q}_{1}$.

With these transformations in hand, we can now proceed to constrain the tunneling matrix coefficients $T_{Q}$. We assume that the tunneling has a non-zero spatial average, which requires the $\boldsymbol{Q}=\mathbf{0}$ term to be non-zero. The matrix $\mathrm{T}_{\mathbf{0}}$ is constrained by the $C_{2} T$ and $R_{y}$ symmetries. Specifically, they imply the two conditions:

$$
\begin{aligned}
C_{2} T: & \mathrm{T}_{0}=\tau^{x} \mathrm{~T}_{0}^{*} \tau^{x}, \\
R_{y}: & \mathrm{T}_{0}=\tau^{x} \mathrm{~T}_{0} \tau^{x} .
\end{aligned}
$$

The most general two-dimensional matrix satisfying Eqs. (21) is

$$
\mathrm{T}_{0}=u \mathbb{I}+w \tau^{x}=u \mathbb{I}+w\left(\tau^{+}+\tau^{-}\right),
$$

where $u$ and $w$ are real parameters. We will determine their physical significance shortly.

Now we address the other $\boldsymbol{Q}$. We expect that tunneling decays for large $|\boldsymbol{Q}|$. Therefore, we will derive a minimal description which assumes the only other non-zero $T_{Q}$ are those which are required to be non-zero to be consistent with symmetry. This is accomplished by applying the condition of $C_{3}$ invariance, which relates other Fourier coefficients of $T$ to $T_{0}$. Invariance under $C_{3}$ (as given in Eq. (19)) of the full tunneling term in Eq. (14) gives

$$
\mathrm{T}(\boldsymbol{u})=e^{-i \boldsymbol{Q}_{1} \cdot \boldsymbol{u}} e^{-2 \pi i \tau^{z} / 3} \mathrm{~T}\left(\mathrm{O}_{3}^{-1} \boldsymbol{u}\right) e^{2 \pi i \tau^{z} / 3}
$$

Now we put the Fourier expansion of Eq. (16) into Eq. (23), and equate coefficients on both sides. This implies

$$
\mathrm{T}_{\mathbf{Q}^{\prime}}=e^{-2 \pi i \tau^{z} / 3} \mathrm{~T}_{\mathbf{Q}} e^{2 \pi i \tau^{z} / 3}, \quad \mathbf{Q}^{\prime}=\mathrm{O}_{3} \mathbf{Q}-\mathbf{Q}_{1} .
$$


Eq. (24), when applied on an initial $T_{Q}$ on the right hand side, generates another $T_{Q^{\prime}}$ on the left, with $\mathbf{Q}^{\prime}=\mathrm{O}_{3} \mathbf{Q}-\mathbf{Q}_{\mathbf{1}}$. We iterate this relation starting with $\mathbf{Q}=\mathbf{0}$ and generate two further Fourier coefficients before the iteration closes.

The result is

$$
\mathrm{T}_{j} \equiv T_{-Q_{j}}=u \mathbb{I}+w\left(\bar{\zeta}^{j} \tau^{+}+\zeta^{j} \tau^{-}\right), \quad j=0,1,2,
$$

where

$$
\zeta=e^{2 \pi i / 3}, \quad \bar{\zeta}=\zeta^{*}=\frac{1}{\zeta}=e^{-2 \pi i / 3},
$$

and

$$
Q_{0}=0, \quad Q_{1}=\frac{4 \pi}{3 a}\left(\begin{array}{c}
\sqrt{3} / 2 \\
1 / 2
\end{array}\right), \quad Q_{2}=\frac{4 \pi}{3 a}\left(\begin{array}{c}
\sqrt{3} / 2 \\
-1 / 2
\end{array}\right)
$$

The three wavevectors $\boldsymbol{Q}_{j}, j=0,1,2$ form an equilateral triangle of reciprocal lattice points whose centroid is the $K$ point (see Fig. 1).

Now we can assign physical significance to $u$ and $w$ by considering some special cases. Suppose we take $\boldsymbol{u}_{l}=\mathbf{0}$, which corresponds to an AA bilayer. We have

$$
\mathrm{T}(\boldsymbol{u})=\sum_{j} \mathrm{~T}_{j} e^{-i \mathbf{Q}_{j} \cdot \boldsymbol{u}} \underset{\boldsymbol{u}=\mathbf{0}}{\longrightarrow} \sum_{j} \mathrm{~T}_{j}=3 u \mathbb{I} .
$$

In the final result, we used the fact that $\sum_{j} \zeta^{j}=\sum_{j} \bar{\zeta}^{j}=0$. Eq. (28) agrees with the simple physical expectation that we just have diagonal sublattice hopping $t^{\prime}$ in this configuration. Hence we conclude that

$$
u=\frac{t_{A A}^{\prime}}{3},
$$

where $t_{A A}^{\prime}$ is the interlayer hopping for an AA region. Now consider $\boldsymbol{u}=(0, a)$, which corresponds to $\mathrm{AB}$ stacking. We see from Eq. (27) that $e^{-i \mathbf{Q}_{j^{\prime}} \cdot \mathbf{u}}=\bar{\zeta}^{j}$. Then we have

$$
\mathrm{T}(\boldsymbol{u})=\sum_{j} \mathrm{~T}_{j} e^{-i \boldsymbol{Q}_{j} \cdot \boldsymbol{u}} \underset{\boldsymbol{u}=(0, a)}{\longrightarrow} \sum_{j} \mathrm{~T}_{j} \bar{\zeta}^{j}=3 w \tau^{-} .
$$

This is again consistent with expectations: interlayer hopping happens only from sublattice B on layer 1 to sublattice A on layer 2, or vice versa. Hence we see that

$$
w=\frac{t_{A B}^{\prime}}{3} .
$$

We have allowed for the inter-layer hopping $t_{A B}^{\prime}$ to be different in the $\mathrm{AB}$ regions from that in the AA ones, though this difference is expected to be small.

We have now fully determined all the terms in the continuum description, by combining Eq. (9), Eq. (11), and Eq. (14), which are now fully specified. The result is given explicitly for clarity in Eq. (39) of the Conclusion.

\subsection{Application to a rigid twist}

To connect to the continuum model of BM, we specialize to a rigid twist, and evaluate the general result for the case

$$
\boldsymbol{u}_{1}=-\boldsymbol{u}_{2}=\frac{\theta}{2} \hat{\boldsymbol{z}} \times \boldsymbol{x} .
$$

For such a pure rotation, the strain-induced gauge field vanishes, $\mathcal{A}_{l}=\mathbf{0}$, and there is zero compression so $\boldsymbol{\nabla} \cdot \boldsymbol{u}_{l}=0$ as well. The $K$-point shift term becomes

$$
\boldsymbol{K} \cdot \partial_{\mu} \boldsymbol{u}_{1}=-\boldsymbol{K} \cdot \partial_{\mu} \boldsymbol{u}_{2}=\mp \frac{\theta K}{2} \hat{\boldsymbol{y}} \equiv \mp \frac{k_{\theta}}{2} \hat{\boldsymbol{y}}
$$


where $k_{\theta}=\theta K$. The displacement gradient itself is

$$
\frac{\partial u_{\mu}^{1}}{\partial x_{v}}=-\frac{\partial u_{\mu}^{2}}{\partial x_{v}}=-\frac{\theta}{2} \epsilon_{\mu \nu} .
$$

Most interestingly, the term in the exponential in the second line of Eq. (39) becomes

$$
\boldsymbol{Q}_{j} \cdot\left(\boldsymbol{u}_{1}-\boldsymbol{u}_{2}\right)=\theta \boldsymbol{Q}_{j} \cdot \hat{\boldsymbol{z}} \times \boldsymbol{x}=-\theta \hat{\boldsymbol{z}} \times \boldsymbol{Q}_{j} \cdot \boldsymbol{x} .
$$

We see that this immediately produces the wavevectors

$$
\boldsymbol{q}_{j}=-\theta \hat{\boldsymbol{z}} \times \boldsymbol{Q}_{j} .
$$

The two non-zero vectors $\boldsymbol{q}_{1}, \boldsymbol{q}_{2}$ are two basis vectors of the reciprocal lattice of the moiré pattern! Putting this all together, we can write the Hamiltonian for the rigid twist as

$$
\begin{aligned}
H_{K, \theta}=\int d^{2} \boldsymbol{x}\left\{\psi_{1}^{\dagger}\left[-i v \tau\left(\frac{\theta}{2}\right) \cdot \boldsymbol{\nabla}-\frac{v k_{\theta}}{2} \tau^{y}\right] \psi_{1}+\psi_{2}^{\dagger}\left[-i v \tau\left(-\frac{\theta}{2}\right) \cdot \boldsymbol{\nabla}+\frac{v k_{\theta}}{2} \tau^{y}\right] \psi_{2}\right. \\
\left.+\sum_{j}\left[e^{-i \boldsymbol{q}_{j} \cdot \boldsymbol{x}} \psi_{2}^{\dagger} \mathrm{T}_{j} \psi_{1}+\text { h.c. }\right]\right\} .
\end{aligned}
$$

Here we defined rotated Pauli matrices

$$
\tau^{\mu}(\theta)=\tau^{\mu}-\theta \epsilon_{\mu \nu} \tau^{\nu}
$$

The result is in perfect agreement with BM.

\section{Conclusion}

We provided a simple real space derivation of a full continuum model for bilayer graphene in the presence of small displacement gradients. Combining all three contributions discussed in Sec. 3 gives the full continuum band Hamiltonian for arbitrary small displacement gradients for the $K$ valley. It is

$$
\begin{aligned}
H_{K}\left[\boldsymbol{u}_{1}, \boldsymbol{u}_{2}\right]=\int d^{2} \boldsymbol{x}\{ & \sum_{l} \psi_{l}^{\dagger}\left[-i v\left(\tau^{\mu}+\frac{\partial u_{\mu}^{l}}{\partial x_{v}} \tau^{\nu}\right) \frac{\partial}{\partial x_{\mu}}+v\left(\boldsymbol{K} \cdot \partial_{\mu} \mathbf{u}_{l}+\mathcal{A}_{l}\right) \tau^{\mu}\right] \psi_{l} \\
& \left.+\sum_{j}\left[e^{-i \boldsymbol{Q}_{j} \cdot\left(\boldsymbol{u}_{1}-\boldsymbol{u}_{2}\right)} \psi_{2}^{\dagger} \boldsymbol{T}_{j} \psi_{1}+\text { h.c. }\right]\right\} .
\end{aligned}
$$

We emphasize that Eq. (39) is derived under the assumption that $\partial_{\mu} \boldsymbol{u}_{l} \ll 1$ and $t^{\prime} \ll t$, but otherwise $\boldsymbol{u}_{l}$ may have arbitrary spatial dependence. As shown in Sec. 3.4 it exactly recovers the BM model for the case of a rigid twist. Eq. (39) goes far beyond this to enable treatment of not only twisting, but also uniform and non-uniform strains. It also ought to be sufficient to understand the coupling of low energy phonons (those derived from the acoustic modes of the original graphene layers) to the bilayer - one needs only to add dynamics to the displacement fields. Finally, Eq. (39) indeed is capable of describing all three of these effects together, simply by taking

$$
\boldsymbol{u}_{l}=(3-2 l) \frac{\theta}{2} \hat{\boldsymbol{z}} \times \boldsymbol{x}+\hat{\mathbf{u}}_{l}, \quad l=1,2,
$$

where $\hat{\boldsymbol{u}}_{l}$ represents the strain and/or phonons. For example, this is a natural point of departure to discuss the effects of twist angle inhomogeneity in twisted bilayer graphene, as there is no need to assume random strains are small compared to the displacement gradient comprising the twist. Hopefully this formulation will have pedagogical value and find useful applications. 


\section{Acknowledgements}

I thank the organizers and students of the EPICQUR summer school for stimulating me to complete this derivation, and for Lucile Savary for helpful advice on the manuscript. This work was supported by the DOE, Office of Science, Basic Energy Sciences under Award No. DE-FG02-08ER46524.

\section{References}

[1] R. Bistritzer and A. H. MacDonald, Moiré bands in twisted double-layer graphene, Proc. Natl. Acad. Sci. 108, 12233 (2011), doi:10.1073/pnas.1108174108.

[2] Y. Cao et al., Correlated insulator behaviour at half-filling in magic-angle graphene superlattices, Nature 556, 80 (2018), doi:10.1038/nature26154.

[3] Y. Cao, V. Fatemi, S. Fang, K. Watanabe, T. Taniguchi, E. Kaxiras and P. Jarillo-Herrero, Unconventional superconductivity in magic-angle graphene superlattices, Nature 556, 43 (2018), doi:10.1038/nature26160.

[4] M. Yankowitz, S. Chen, H. Polshyn, Y. Zhang, K. Watanabe, T. Taniguchi, D. Graf, A. F. Young and C. R. Dean, Tuning superconductivity in twisted bilayer graphene, Science 363, 1059 (2019), doi:10.1126/science.aav1910.

[5] Y. Choi et al., Electronic correlations in twisted bilayer graphene near the magic angle, Nat. Phys. (2019), doi:10.1038/s41567-019-0606-5.

[6] A. L. Sharpe, E. J. Fox, A. W. Barnard, J. Finney, K. Watanabe, T. Taniguchi, M. A. Kastner and D. Goldhaber-Gordon, Emergent ferromagnetism near three-quarters filling in twisted bilayer graphene, Science 365, 605 (2019), doi:10.1126/science.aaw3780.

[7] H. Yoo et al., Atomic and electronic reconstruction at the van der waals interface in twisted bilayer graphene, Nat. Mater. 18, 448 (2019), doi:10.1038/s41563-019-0346-z.

[8] X. Lu et al., Superconductors, orbital magnets, and correlated states in magic angle bilayer graphene (2019), arXiv:1903.06513.

[9] A. Kerelsky, L. J. McGilly, D. M. Kennes, L. Xian, M. Yankowitz, S. Chen, K. Watanabe, T. Taniguchi, J. Hone, C. Dean, A. Rubio and A. N. Pasupathy, Maximized electron interactions at the magic angle in twisted bilayer graphene, Nature 572, 95 (2019), doi:10.1038/s41586-019-1431-9.

[10] K. Kim, A. DaSilva, S. Huang, B. Fallahazad, S. Larentis, T. Taniguchi, K. Watanabe, B. J. LeRoy, A. H. MacDonald and E. Tutuc, Tunable moiré bands and strong correlations in small-twist-angle bilayer graphene, Proc. Natl. Acad. Sci. 114, 3364 (2017), doi:10.1073/pnas.1620140114.

[11] N. N. Nam and M. Koshino, Lattice relaxation and energy band modulation in twisted bilayer graphene, Phys. Rev. B 96, 075311 (2017), doi:10.1103/PhysRevB.96.075311.

[12] A. Uri et al., Mapping the twist angle and unconventional landau levels in magic angle graphene (2019), arXiv:1908.04595. 
[13] S. Xu et al., Giant oscillations in a triangular network of one-dimensional states in marginally twisted graphene, Nat. Comm. 10, 4008 (2019), doi:10.1038/s41467-01911971-7.

[14] Z. Bi, N. F. Q. Yuan and L. Fu, Designing flat bands by strain, Phys. Rev. B 100, 035448 (2019), doi:10.1103/PhysRevB.100.035448.

[15] B. Lian, Z. Wang and B. A. Bernevig, Twisted bilayer graphene: A phonon-driven superconductor, Phys. Rev. Lett. 122, 257002 (2019), doi:10.1103/PhysRevLett.122.257002.

[16] F. Wu, A. H. MacDonald and I. Martin, Theory of phonon-mediated superconductivity in twisted bilayer graphene, Phys. Rev. Lett. 121, 257001 (2018), doi:10.1103/PhysRevLett.121.257001.

[17] Y. W. Choi and H. J. Choi, Strong electron-phonon coupling, electron-hole asymmetry, and nonadiabaticity in magic-angle twisted bilayer graphene, Phys. Rev. B 98, 241412 (2018), doi:10.1103/PhysRevB.98.241412.

[18] F. Wu, E. Hwang and S. Das Sarma, Phonon-induced giant linear-in-T resistivity in magic angle twisted bilayer graphene: Ordinary strangeness and exotic superconductivity, Phys. Rev. B 99, 165112 (2019), doi:10.1103/PhysRevB.99.165112.

[19] P. A. Lee and T. M. Rice, Electric field depinning of charge density waves, Phys. Rev. B 19, 3970 (1979), doi:10.1103/PhysRevB.19.3970.

[20] H. Fukuyama and P. A. Lee, Dynamics of the charge-density wave. i. impurity pinning in a single chain, Phys. Rev. B 17, 535 (1978), doi:10.1103/PhysRevB.17.535.

[21] H. Suzuura and T. Ando, Phonons and electron-phonon scattering in carbon nanotubes, Phys. Rev. B 65, 235412 (2002), doi:10.1103/PhysRevB.65.235412.

[22] A. C. Neto, F. Guinea, N. M. Peres, K. S. Novoselov and A. K. Geim, The electronic properties of graphene, Rev. Mod. Phys. 81, 109 (2009), doi:10.1103/RevModPhys.81.109. 